\title{
XBP1s-Mediated Endoplasmic Reticulum Proteostasis Network Enhancement Can Selectively Improve Folding and Secretion of an Osteogenesis Imperfecta-Causing Collagen-I Variant
}

Andrew S. DiChiara, ${ }^{1}$ Ngoc-Duc Doan, ${ }^{1}$ Agata A. Bikovtseva, ${ }^{1}$ Lynn Rowley, ${ }^{2}$ Vincent L. Butty, ${ }^{3}$ MaryAnn E. Weis, ${ }^{4}$ David R. Eyre, ${ }^{4}$ Shireen R. Lamandé, $, 2,5$ John F. Bateman, ${ }^{2,5}$ and Matthew D. Shoulders ${ }^{1, *}$

1Department of Chemistry, Massachusetts Institute of Technology, Cambridge, MA, USA; ${ }^{2}$ Murdoch Children's Research Institute, Victoria, Australia; ${ }^{3}$ BioMicro Center, Massachusetts Institute of Technology, Cambridge, MA, USA; ${ }^{4}$ Department of Orthopaedics and Sports Medicine, University of Washington, Seattle, WA, USA; ${ }^{5}$ Department of Paediatrics, University of Melbourne, Melbourne, Victoria, Australia

${ }^{*}$ To whom correspondence may be addressed:

Matthew D. Shoulders

Department of Chemistry

Massachusetts Institute of Technology

77 Massachusetts Ave., 16-573A

Cambridge, MA 02139

Phone: (617) 452-3525

Email: mshoulde@mit.edu 


\section{ABSTRACT}

Osteogenesis imperfecta $(\mathrm{OI})$ is typically caused by autosomal dominant mutations in genes encoding collagen type-I, most commonly resulting in Gly $\rightarrow$ Ser triple-helical domain substitutions that disrupt collagen folding and/or stability. Here, we test the hypothesis that upregulating the endoplasmic reticulum (ER) proteostasis network via the unfolded protein response (UPR) can improve the folding and secretion of the clinically severe, prototypical OI-causing COL1A1 p.G425S collagen- $\alpha 1(\mathrm{I})$ variant. We first show that small molecule stressors that globally activate the UPR severely ablate collagen-I secretion from both G425S Cola1(I)- and wild-type (WT) Cola1(I)-expressing primary fibroblasts. In contrast, stress-independent, specific induction of just the UPR's XBP1s transcriptional response enhances collagen-I secretion from G425S Col $\alpha 1(\mathrm{I})$ patient primary fibroblasts up to $\sim 300 \%$ of basal levels. Notably, the effect is selective - collagen-I secretion from WT Cola1(I)-expressing healthy donor primary fibroblasts is unaltered by XBP1s. XBP1s pathway activation appears to post-translationally enhance the folding/assembly and secretion of G425S Cola1(I), as only modest impacts on collagen-I transcription or synthesis are observed. Consistent with this notion, we find that the stable, triple-helical collagen-I secreted by XBP1s-activated G425S $\alpha 1$ (I) patient fibroblasts includes a higher proportion of the mutant $\alpha 1(\mathrm{I})$ polypeptide than the collagen-I secreted under basal ER proteostasis conditions. These results highlight the potential for ER proteostasis network modulation to improve mutant collagen proteostasis in the collagenopathies, motivating further investigation of the effect's generality, underlying mechanism, and potential therapeutic benefits. 


\section{INTRODUCTION}

Mutations that result in dysregulated collagen proteostasis, defined as an inability of cells to handle and produce appropriate quantities of folded and functional collagen, cause an array of diseases known as the collagenopathies ${ }^{1,2}$. The collagenopathies have divergent clinical phenotypes, ranging from mild to severe, depending on the specific mutation and the type of collagen involved. The prototypical collagenopathy, osteogenesis imperfecta $(\mathrm{OI})$, is characterized by symptoms such as bone fragility, reduced bone mass, and short stature $^{3}$. As is also true for the other collagenopathies, current OI therapies remain inadequate for alleviating both skeletal and non-skeletal manifestations of the disease.

OI is most often caused by autosomal dominant mutations in the COL1A1 or COL1A2 genes that encode the procollagen- $\alpha 1(\mathrm{I})$ and procollagen- $\alpha 2(\mathrm{I})$ strands of the 2:1 pro $\alpha 1(\mathrm{I})$ :pro $\alpha 2(\mathrm{I})$ heterotrimeric procollagen molecule (procollagen-I refers to collagen-I prior to the extracellular cleavage of its N- and C-propeptides) $)^{3}$. The most common class of Ol-causing mutations are missense mutations causing substitution of various conserved Gly residues within the central, functionally critical triple-helical domain of collagen-I ${ }^{4}$. A Gly residue is required at every third position within the triple-helical domain of mature collagen polypeptides, because it is the only amino acid small enough to be accommodated in the interior of the sterically occluded triple helix ${ }^{5}$. Substitution of Gly with any other amino acid creates a steric "bump" in the folded triple helix, disrupts the stabilizing ladder of interstrand $\mathrm{N}-\mathrm{H}_{(\mathrm{Gly})} \cdots \mathrm{O}=\mathrm{C}_{(\text {Xaa) }}$ hydrogen bonds, and delays triple-helix folding (Figure 1) $)^{5,6}$.

Ol-causing mutations can engender at least three defects that pathologically disrupt collagen proteostasis: (1) Non-functional collagen may be allowed to escape the cell instead of being subjected to quality control. Secreted, non-functional collagens may cause bone defects ${ }^{7-9}$ or disrupt essential extracellular matrix proteinprotein and/or protein-cell interactions ${ }^{4,10,11}$. (2) Collagen-I mutations could result in insufficient production of functional collagen-I, owing to hyperactive quality control and inefficient folding. A reduction in functional collagen-I production itself can be damaging ${ }^{12,13}$. Slow collagen-I folding and secretion often also results in overhydroxylation of triple-helical lysine residues ${ }^{14-16}$, which may result in excessive collagen-I crosslinking, hyperO-glycosylation, and consequent pathologic structural defects in bone and other tissues ${ }^{17}$. (3) Misfolding or slow-folding collagen-I could overwhelm the endoplasmic reticulum (ER) proteostasis network, resulting in intracellular collagen accumulation and chronic cell stress, likely promoting the apoptosis of collagen-I producing 
osteoblasts and thereby reducing bone health ${ }^{18-21}$. The resultant ER dysfunction could also lead to a net decrease in collagen-I production that exceeds the consequences of haploinsufficiency mutations in collagen-I (e.g., premature stop codons). Haploinsufficiency mutations reduce the net synthesis of collagen-I but do not create a folding challenge for the ER and cause only mild $\mathrm{OI}^{3,12}$.

Distinctive OI mutations likely lead to disease by various combinations of one or more of these mechanisms. The integral role of dysregulated collagen proteostasis in all these mechanisms highlights the need to develop strategies that can alleviate such defects, if possible ${ }^{1,2,19}$. The endogenous cellular signaling pathway typically responsible for addressing defects in ER proteostasis is the unfolded protein response (UPR) ${ }^{22,23}$. The UPR consists of three distinct arms, ATF6, IRE1-XBP1s, and PERK-ATF4, that cooperate to resolve ER proteostasis defects by transcriptionally remodeling the ER's chaperone and quality control network while simultaneously reducing translation to lower the client protein load on the ER. The predominant function of the ATF6 and IRE1-XBP1s UPR arms is to restore proteostasis by transcriptionally upregulating ER chaperones and quality control machinery through the activity of the cleaved ATF6(1-373) and XBP1s transcription factors. The PERK UPR arm regulates ER client protein translation and, if chronically activated, ultimately causes cellular apoptosis.

Misfolding- or aggregation-causing mutations in ER proteins can, at least in some cases, lead directly to UPR induction ${ }^{24}$. The resulting UPR activation, by reshaping the ER proteostasis network to better support protein folding and production, can prove beneficial to help address the underlying proteostasis defect, although long-term chronic ER stress is problematic ${ }^{23,25}$. Regardless, UPR activation by the common diseasecausing, triple-helical Gly substitutions in collagen- $\alpha 1(\mathrm{I})$ and collagen- $\alpha 2(\mathrm{I})(\alpha 1(\mathrm{I})$ and $\alpha 2(\mathrm{I})$, respectively) is not generally observed $^{18,19,26-28}$. The lack of UPR activation despite the presence of a large quantity of a misfolding client protein may reflect an inability of the ER's protein misfolding sensors to detect accumulation of an atypical misfolded fibrous protein, rather than a globular protein with a hydrophobic core.

The absence of a prototypical UPR in most collagenopathies could present an opportunity for therapeutic intervention. Specifically, there is still the possibility that enhancing the ER proteostasis network via artificial UPR activation could prove beneficial for resolving collagen-I proteostasis defects, for example by improving 
folding and trafficking of OI variants. Indeed, in a number of protein misfolding-related disease contexts, genetic or pharmacologic UPR modulation has been shown to address underlying proteostasis defects ${ }^{29-34}$. For example, the UPR-mediated ER proteostasis network upregulation induced by treatment with chemical ER stressors enhances the folding and trafficking of misfolding-prone lysosomal enzyme variants to the lysosome, where they are sufficiently active to rescue lysosome function ${ }^{35,36}$. This enhanced trafficking also relieves ER dysfunction caused by the poorly trafficked enzyme variants.

Here, we show that remodeling the ER proteostasis network via forced expression of the UPR's XBP1s transcription factor improves the folding and secretion of collagen-I by OI patient primary fibroblasts. XBP1s selectively enhances mutant collagen-I proteostasis, while having no impact on the folding and trafficking of collagen-I by wild-type (WT) $\alpha 1$ (I)-expressing primary fibroblasts. The effect can be attributed to a post-translational impact on mutant collagen-I folding, assembly, and trafficking, as XBP1s only minimally influences collagen-I transcription or translation. Consistent with this observation, we find that collagen-I molecules secreted from XBP1s-treated primary OI patient fibroblasts exhibit increased incorporation of G425S $\alpha 1(\mathrm{I})$ into secreted, stable triple helices. These observations provide proof-of-principle for the notion that pharmacologic targeting of the ER proteostasis network can address disease-causing collagen proteostasis defects. 


\section{MATERIALS AND METHODS}

\section{Cell Culture}

WT $\alpha 1$ (I)-expressing (GM05294; healthy donor) or G425S (numbering from the first residue of the Npropeptide; this mutation corresponds to G247S when numbering is started at the first glycine of the triple helix) $\alpha 1$ (I)-expressing (GM05747; OI patient) primary fibroblasts were obtained from Coriell Cell Repository and cultured in complete MEM (Corning) supplemented with 15\% fetal bovine serum (FBS; Corning), 100 IU penicillin (Corning), $100 \mu \mathrm{g} / \mathrm{mL}$ streptomycin (Corning), and $2 \mathrm{mM} \mathrm{L-glutamine} \mathrm{(Corning).} \mathrm{For} \mathrm{sub-culturing,} \mathrm{cells}$ were trypsinized and resuspended in ample fresh media, collected by centrifugation, and resuspended in complete fresh media to count and then plate cells for experiments. Cells were counted using the Countess II FL (Life Technologies). Collagen-I production was induced using $200 \mu \mathrm{M}$ sodium ascorbate (Amresco). Cell viability assessment was performed using the CellTiter-Glo® assay (Promega) according to the manufacturer's instructions.

\section{Cell Lysis, SDS-PAGE, and Immunoblotting}

After trypsinization and neutralization in complete media, intact cells were collected by centrifugation, washed with $1 \times$ PBS, and then lysed in radio-immunoprecipitation assay buffer (RIPA) containing 150 mM sodium chloride, $50 \mathrm{mM}$ Tris- $\mathrm{HCl}$ at $\mathrm{pH}$ 7.5, 1\% Triton-X 100 (Integra), 0.5\% sodium dodecyl sulfate, $0.1 \%$ sodium deoxycholate, protease inhibitor tablets (Pierce), and $1 \mathrm{mM}$ phenylmethylsulfonyl fluoride. Cells were lysed on ice for $20 \mathrm{~min}$, the lysate was cleared by centrifugation at $16,800 \mathrm{rpm}$ at $4{ }^{\circ} \mathrm{C}$, and then protein concentration in the supernatant was quantified and normalized prior to separation under reducing conditions on 8\% SDS-PAGE. Identical volumes of media were also collected and used directly for SDS-PAGE. For immunoblotting, proteins were transferred to nitrocellulose membranes using the TransBlot Turbo system (BioRad) using transfer buffer at pH 9.2 containing $48 \mathrm{mM}$ Tris, $39 \mathrm{mM}$ glycine, $0.1 \%$ SDS, and $20 \%$ ethanol. Membranes were blocked in 5\% milk and then probed with the indicated primary antibodies diluted in $5 \%$ bovine serum albumin (VWR). Blots were imaged after incubation with appropriate primary and secondary antibodies by scanning on an Odyssey IR imager (LI-COR). Antibodies used were: rabbit anti- $\alpha 1$ (I) [LF68] (Kerafast ENH018-FP) raised against the C-telopeptide of the $\alpha 1(\mathrm{I})$ chain (Figures: 2a, 3, and S2); rabbit antiCola1(I) (MyBioSource MBS502153) raised against the C-telopeptide of the $\alpha 1$ (I) chain (Figure: 2c); rabbit 
anti- $\alpha 2(\mathrm{I})$ (Sigma SAB4500363) raised against human $\alpha 2(\mathrm{I})$ triple-helical domain-derived peptides (Figures: 2a,

3, 5a, and S2). Secondary antibodies were obtained from LiCor Biosciences: 800CW goat anti-rabbit, 800CW goat anti-mouse, 680LT goat anti-rabbit, 680LT goat anti-mouse.

\section{Adenovirus Transductions}

The ViraPower ${ }^{\mathrm{TM}}$ Adenoviral Gateway ${ }^{\mathrm{TM}}$ system was used to generate replication-incompetent adenoviruses encoding either XBP1s, eGFP, DHFR.ATF6(1-373), ${ }^{31}$ or DHFR.YFP. Viruses were titered using the Adeno-X qPCR Titration kit (Clontech), according to the manufacturer's instructions. WT $\alpha 1(\mathrm{I})-$ or G425S $\alpha 1(\mathrm{I})-$ expressing primary fibroblasts $\left(1.0 \times 10^{5}\right.$ cells $)$ were plated the day before transduction in a 6 -well plate. $24 \mathrm{~h}$ post-plating, media was replaced and cells were treated with the indicated adenovirus at an experimentally optimized multiplicity of infection determined for each batch of adenovirus used. Following $24 \mathrm{~h}$ of incubation with virus, media was replaced. $48 \mathrm{~h}$ post-media change, the media was replaced with fresh media supplemented with $200 \mu \mathrm{M}$ Asc. Following $\sim 24 \mathrm{~h}$ of incubation with Asc, cells were harvested by trypsinization. For experiments involving DHFR.ATF6(1-373) or DHFR.YFP, WT Col $\alpha 1(\mathrm{I})$-expressing or G425S $\alpha 1(\mathrm{I})$-expressing primary fibroblasts $\left(2.0 \times 10^{5}\right.$ cells $)$ were plated the day before transduction in a 6 -well plate. $24 \mathrm{~h}$ post-plating, the media was replaced and supplemented with DHFR.ATF6(1-373) or DHFR.YFP adenoviral supernatant at an experimentally optimized MOI yielding TMP-dependent activation of the ATF6 arm of the UPR. Following 24 $\mathrm{h}$ of incubation with virus, cells were split and then supplemented with media containing $10 \mu \mathrm{M}$ TMP or vehicle. $48 \mathrm{~h}$ post-media change or split, media was removed and replaced with $1 \mathrm{~mL}$ of fresh media supplemented with $200 \mu \mathrm{M}$ ascorbate. Media and cells were harvested for downstream analysis after $\sim 18 \mathrm{~h}$.

\section{Quantitative RT-PCR}

Relative mRNA expression levels of genes of interest were assessed by quantitative RT-PCR. Cells were harvested by trypsinization and washed with PBS. Total RNA was extracted using the Omega RNA Purification Kit. RNA concentrations were quantified and normalized to $1 \mu \mathrm{g}$ total RNA for cDNA reverse transcription. cDNA was synthesized in a BioRad Thermocycler using the Applied Biosystems reverse transcriptase cDNA kit. KAPA BioSystems Sybr Fast qPCR master mix, appropriate primers (Sigma Aldrich; see Table S3), and cDNA were then used for amplification in a Light Cycler 480 II real-time PCR Instrument. Primer integrity 
was assessed by thermal melt to ensure homogeneity. Transcripts were normalized to the housekeeping gene RPLP2. Standard deviation for $n=3$ is shown in plots.

\section{RNA-Seq}

Cells were transduced with Ad.GFP or Ad.XBP1s and then treated with Asc as described above. Following treatment with Asc, cells were harvested by trypsinization, washed with PBS, and total RNA was extracted using the Omega RNA Purification Kit. RNA samples were quantified and quality assessed using an Advanced Analytical Fragment Analyzer. Initial steps in library preparation were then performed on a Tecan EVO150. $10 \mathrm{ng}$ of total RNA was used for library preparation. 3'-DGE-custom primers 3V6NEXT-bmc\#1-12 were added to a final concentration of $1 \mu \mathrm{M}\left(5^{\prime}-/ 5\right.$ Biosg/ACACTCTTTCCCTACACGACGCTCTTCCGATCT[BC 6 ] $\mathrm{N}_{10} \mathrm{~T}_{30} \mathrm{VN}-3^{\prime}$ where 5Biosg = 5'- biotin, [BC6] = 6 bp barcode specific to each sample/well, N10 = Unique Molecular Identifiers, Integrated DNA Technologies). After addition of the oligonucleotides, Maxima H Minus RT was added per the manufacturer's recommendations with the template-switching oligo 5V6NEXT (10 $\mu \mathrm{M}$, [5V6NEXT: 5'-iCiGiCACACTCTTTCCCTACACGACGCrGrGrG-3' where iC: iso-dC, iG: iso-dG, rG: RNA G]) followed by incubation at $42{ }^{\circ} \mathrm{C}$ for 90 min and inactivation at $80{ }^{\circ} \mathrm{C}$ for 10 min. Following the template-switching reaction, cDNA from 12 wells containing unique well identifiers were pooled together and cleaned using RNA Ampure beads at 1.0x. cDNA was eluted with $17 \mu \mathrm{L}$ of water followed by digestion with exonuclease $\mathrm{I}$ at $37^{\circ} \mathrm{C}$ for $30 \mathrm{~min}$, and then inactivated at $80^{\circ} \mathrm{C}$ for $20 \mathrm{~min}$. Second strand synthesis and PCR amplification was performed by adding the Advantage 2 Polymerase Mix (Clontech) and the SINGV6 primer (10 pmol, Integrated DNA Technologies 5'-/5Biosg/ACACTCTTTCCCTACACGACGC-3') directly to the exonuclease reaction. 15 cycles of PCR were performed followed by clean-up using regular SPRI beads at $0.6 \times$, and eluted with $20 \mu \mathrm{L}$ of elution buffer. Successful amplification of cDNA was confirmed using a Fragment Analyzer. Illumina libraries were then produced using standard Nextera tagmentation, substituting the P5NEXTPT5-bmc primer (25 $\mu \mathrm{M}$, Integrated DNA Technologies (5'-AATGATACGGCGACCACCGAGATCTACACTCTTTCCCTACACGACGCTCTTCCG ${ }^{*} A^{*} T^{*} C^{*} T^{*}-3^{\prime}$ where * $=$ phosphorothioate bonds) in place of the normal N500 primer. Final libraries were cleaned using SPRI beads at $0.7 \times$ and quantified using a Fragment Analyzer and qPCR before being loaded for paired-end sequencing using the Illumina NextSeq500 in paired-end mode (20/50 nt reads). 
Post-sequencing, quality-control on each of the libraries was performed to assess coverage depth, enrichment for messenger RNA (exon/intron and exon/intergenic density ratios), fraction of rRNA reads and number of detected genes using bespoke scripts. Read pairs were combined into a single Fastq with well/unique molecular identifier (UMI) information concatenated with the second read name. Reads sharing sequence and UMI information were collapsed into single exemplars. Sequences were aligned against the human genome GRCh38/hg38 ENSEMBL 89 using STAR ${ }^{37}$, with parameters --runThreadN 8 --runMode alignReads --outFilterType BySJout --outFilterMultimapNmax 20 --alignSJoverhangMin 8 --alignSJDBoverhangMin 1 --outFilterMismatchNmax 999 --alignIntronMin 10 --alignIntronMax 1000000 --alignMatesGapMax 1000000 --outSAMtype BAM SortedByCoordinate --quantMode TranscriptomeSAM, pointing to a Suffix Array assembly containing exon-exon junctions for 75 nucleotide reads. Gene expression was estimated based on reads mapping near the 3' end of transcripts using ESAT ${ }^{38}$ with the following parameters -task score $3 p$-alignments \$sample_list -wLen 50 -wExt 5000 -wOlap 0 -sigTest 0.01 -multimap ignore, pointing to a refseq-based gene annotation (hg38, as downloaded from the UCSC Genome Browser) and gene-level data were used for downstream analyses.

Differential expression was performed in the R statistical environment ( $R$ v. 3.4.0) using Bioconductor's DESeq2 package on all annotated genes ${ }^{39}$. Dataset parameters were estimated using the estimateSizeFactors(), and estimateDispersions() functions. Read counts across conditions were modeled based on a negative binomial distribution and a Wald test was used to test for differential expression (nbinomWaldtest(), all packaged into the DESeq() function), using the treatment type as a contrast and the replicate of origin as a factor. Fold-changes, $p$-values and Benjamin-Hochberg-adjusted $p$-values $(\mathrm{BH})$ are reported for each gene. Regularized fold-changes were calculated using the lfcShrink() function in "normal" mode.

GSEA was performed to compare genes upregulated upon XBP1s induction in WT vs G425S $\alpha 1(\mathrm{I})$ primary fibroblast cells. Differential expression results from DESeq2 were retrieved, and the "stat" column was used to pre-rank genes for GSEA. Briefly, these "stat" values reflect the Wald's test performed on read counts as modeled by DESeq2 using the negative binomial distribution. Genes that were not expressed were excluded from the analysis. GSEA (desktop version, v3.0) 40, 41 was run in the pre-ranked mode against MsigDB 
7.0 C5 (gene ontology) sets, using the official gene symbol as the key, with a weighted scoring scheme, normalizing by meandiv, with gene sets between 10 and 2000 genes (9438 gene sets retained for C5). 5000 permutations were run for $p$-value estimation. GSEA enrichment plots were replotted using a modified version of the ReplotGSEA.R script.

\section{Metabolic Labeling}

$72 \mathrm{~h}$ post-transduction with Ad.GFP or Ad.XBP1s adenoviruses, media was removed from WT $\alpha 1$ (I) or G425S $\alpha 1$ (I) primary fibroblasts and replaced with fresh media containing $200 \mu \mathrm{M}$ ascorbate. $2 \mathrm{~h}$ later, media was replaced with fresh media containing $200 \mu \mathrm{M}$ ascorbate and $100 \mathrm{mCi} / \mathrm{mL}$ of ${ }^{35} \mathrm{~S}-\mathrm{Cys} /$ Met (Perkin Elmer) for 10 min. Following labeling, media was removed, cells were washed $2 \times$ with PBS, and then lysed immediately in the plate using RIPA. Following 20 min of lysis on ice, samples were collected and cell debris was removed by centrifugation at $10,000 \times g$ and $4^{\circ} \mathrm{C}$ for $15 \mathrm{~min}$. Supernatants were collected, split equally into two tubes, and supplemented with $8 \times$ collagenase buffer containing $50 \mathrm{mM}$ HEPES at pH 7.5 and $360 \mu \mathrm{M}$ calcium chloride. Half of the samples were treated with a final concentration of $5 \mu \mathrm{g} / \mathrm{mL}$ bacterial collagenase (Worthington Biochemical) for $3 \mathrm{~d}$ at $37^{\circ} \mathrm{C}$. Samples were then loaded onto homemade $10 \%$ SDS-PAGE gels. Gels were dried, developed for $2 \mathrm{~d}$, and imaged using a phosphor screen and a Typhoon 7800 imager. Band intensities were quantified using ImageQuant TL (GE Healthcare). Experiments were performed in biological triplicate with standard deviation shown.

\section{Secreted Collagen-I Assessment}

WT $\alpha 1(\mathrm{I})$-expressing or G425S $\alpha 1$ (I)-expressing primary fibroblasts were plated in a 6-cm dish, and then transduced $24 \mathrm{~h}$ later with Ad.GFP or Ad.XBP1s. $24 \mathrm{~h}$ post-transduction, media was changed. $72 \mathrm{~h}$ posttransduction, collagen production was induced by treatment with $200 \mu \mathrm{M}$ ascorbate for $24 \mathrm{~h}$. Media was collected, chilled at $4{ }^{\circ} \mathrm{C}$, and treated with $1 \mathrm{mM} \mathrm{PMSF}, 176 \mathrm{mg} / \mathrm{mL}$ ammonium sulfate, and $100 \mathrm{mM}$ Tris- $\mathrm{HCl}$ at $\mathrm{pH} 7.4$ to precipitate the secreted procollagen-I. Samples were mixed end over end overnight at $4{ }^{\circ} \mathrm{C}$, and pelleted by centrifugation at $10,000 \times g$ and $4{ }^{\circ} \mathrm{C}$ for $30 \mathrm{~min}$. Supernatant was removed and the remaining pellet was resolubilized in $400 \mathrm{mM} \mathrm{NaCl}$ and $100 \mathrm{mM}$ Tris- $\mathrm{HCl}$ at $\mathrm{pH}$ 7.4. Collagen-I samples were then aliquoted 
and treated with $0.1 \mathrm{mg} / \mathrm{mL}$ trypsin or $0.2 \mathrm{mg} / \mathrm{mL}$ chymotrypsin (Sigma Aldrich) ${ }^{42}$. Samples were analyzed by SDS-PAGE using an antibody raised against peptides derived from the triple-helical domain of $\alpha 2(\mathrm{I})$.

\section{Mass Spectrometry Analysis of Secreted G425S Cola1(I)}

G425S $\alpha 1(\mathrm{I})$ primary patient fibroblasts $\left(1.0 \times 10^{5}\right.$ cells $)$ were plated in a 6 -well plate and transduced 24 h later with Ad.GFP or Ad.XBP1s. 24 h post-transduction, media was changed. 72 h post-transduction, collagen production was initiated by treating with $200 \mu \mathrm{M}$ ascorbate for $24 \mathrm{~h}$. Media samples were then collected, chilled at $4{ }^{\circ} \mathrm{C}$, and treated with $1 \mathrm{mM}$ PMSF. Samples were acidified to $3 \%$ acetic acid and treated with pepsin $\left(\sim 0.1 \mathrm{mg} / \mathrm{ml}\right.$ and $\sim 1: 10$ pepsin:collagen) for $24 \mathrm{~h}^{43}$. Collagen was then precipitated by addition of sodium chloride to a $0.7-0.9 \mathrm{M}$ final concentration. The resulting collagen was separated on a homemade $6 \%$ SDS-PAGE gel. The gel was stained with Coomassie dye and the $\alpha 1(\mathrm{I})$ band was excised, reduced with DTT, alkylated with iodoacetamide, and trypsinized overnight. The resultant tryptic peptides were analyzed by LC-MS/MS analysis to determine the abundance of the WT $\alpha 1(\mathrm{I})$ - and G425S $\alpha 1(\mathrm{I})$-containing peptides. The difference between the WT $\alpha 1(\mathrm{I})$ and $\mathrm{G} 425 \mathrm{~S} \alpha 1(\mathrm{I})$ peptides was quantified by analyzing the area under the curve of the liquid chromatography trace for each peptide.

\section{Data Availability}

The RNA-Seq primary data were deposited in NCBI's GEO repository under the accession number GSE163812. 


\section{RESULTS}

\section{Global Unfolded Protein Response Activation Exacerbates the OI Collagen-I Secretion Defect in Fibro- blasts Expressing the COL1A1 p.G425S Mutation}

To begin, we compared the collagen-I secretion profile of wild-type (WT) $\alpha 1(\mathrm{I})$ healthy donor primary fibroblasts to that of OI patient primary fibroblasts heterozygous for WT $\alpha 1(\mathrm{I})$ and G425S $\alpha 1(\mathrm{I})$. This common, prototypical OI-causing amino acid substitution replaces a Gly residue within the collagen-I triple-helical domain with a Ser residue, breaking the interstrand hydrogen bond between the triple helix-forming polypeptides and causing severe forms of $\mathrm{OI}$, most commonly the lethal type-II form of $\mathrm{Ol}^{44}$. We observed that, upon ascorbate (Asc) stimulation of collagen production, collagen-I secretion over a $24 \mathrm{~h}$ period was significantly reduced in the heterozygous G425S $\alpha 1$ (I)-expressing fibroblasts compared to WT $\alpha 1(\mathrm{I})$-expressing control fibroblasts (Figure 2a; note that in this and other media immunoblots it is not possible to normalize to a 'housekeeping' protein like actin due to the absence of stable markers in media - instead, we carefully ensured equivalent cell counts, media volumes, and timecourses between samples as described in the Materials and Methods). Similar Ol-associated collagen-I secretion defects have been observed across many OI genotypes ${ }^{15,} 16,45-51$.

qPCR analysis of mRNA levels showed that classic UPR markers, including HSPA5, DNAJB9, and HYOU1, were not upregulated (Figure $\mathbf{2 b}$ ) $^{23}$. Thus, as expected based on prior work with other triple-helical collagen-I Ol-causing variants ${ }^{18,19,26-28}$, the collagen-I secretion defect observed in G425S $\alpha 1$ (I) OI patient fibroblasts did not induce a prototypical UPR.

We hypothesized that this absence of a prototypical UPR actually presents an opportunity for intervention. Specifically, there is still the possibility that enhancement of the ER proteostasis network via artificial UPR activation could prove beneficial for G425S $\alpha 1(\mathrm{I})$ folding and trafficking. To test this hypothesis, we treated WT $\alpha 1(\mathrm{I})$-expressing or G425S $\alpha 1(\mathrm{I})$-expressing primary fibroblasts for $24 \mathrm{~h}$ with low concentrations of the ER stressor thapsigargin $(\mathrm{Tg})$ ranging from 0.3-100 $\mathrm{nM}$. Tg inhibits $\mathrm{Ca}(\mathrm{II})$ flux and acts as a rapid chemical activator of the global UPR, upregulating ER chaperones and quality control machineries ${ }^{52}$. We observed that even the very lowest concentration of Tg that reliably activated the UPR (10.0 nM) either had no effect or caused a strong decrease in collagen-I secretion from not just G425S $\alpha 1(\mathrm{I})$ fibroblasts but also from WT $\alpha 1$ (I) fibroblasts

(Figure 2c; see Figures S1a and S1b for qPCR assessment of UPR activation). This decrease in collagen-I 
secretion was not specific to the use of Tg treatment as a strategy for global UPR activation. A second chemical UPR activator, tunicamycin (Tm; an N-glycosylation inhibitor $\left.{ }^{53}\right)$, also reduced collagen-I secretion from both WT and OI patient primary cell lines even at the lowest concentration $(0.6 \mu \mathrm{g} / \mathrm{mL})$ capable of inducing the UPR (Figure 2c; see Figures S1c and S1d for qPCR assessment of UPR activation).

The finding that global UPR activation negatively impacted collagen-I secretion, while arguably disappointing, is unsurprising. Chemical ER stressors induce the UPR by causing general ER protein misfolding. In some cases, proteostasis defects associated with individual ER client proteins (e.g., lysosomal enzymes ${ }^{35}$ ) can still be addressed by this approach to ER proteostasis network remodeling. More commonly, however, stressmediated induction of the entire UPR, including the PERK arm of the UPR that inhibits protein translation ${ }^{22}$, only exacerbates disease-causing ER proteostasis defects. Thus, the use of non-selective ER stressors was not a viable method for correcting the collagen-I secretion defect in G425S $\alpha 1$ (I) OI patient primary fibroblasts.

\section{Stress-Independent Induction of the XBP1s Transcriptional Pathway Ameliorates the OI Collagen-I Se- cretion Defect}

Whereas global UPR activation using chemical ER stressors is often problematic, a growing body of work suggests there is great promise in the stress-independent induction of individual UPR arms ${ }^{25,29,31-33,54-58 .}$ A number of genetic and/or chemical genetic approaches are now available to force the expression of the active forms of the ATF6 and XBP1s transcription factors, the two arms of the UPR that enhance the expression of ER chaperones and quality control machineries and that are therefore most likely to result in productive ER proteostasis network remodeling ${ }^{56}$. These strategies have shown promise in neurodegenerative disorders ${ }^{32,57}$, ${ }^{58}$, other protein aggregation-related diseases ${ }^{31,54,59}$, and in a variety of additional contexts ${ }^{33,55}$. Arm-specific UPR pathway activation has not, however, been assessed as a strategy to address collagen proteostasis defects in OI or the other collagenopathies.

We hypothesized that activation of either the ATF6 or the IRE1-XBP1s transcriptional pathways could be beneficial in G425S $\alpha 1$ (I) OI patient fibroblasts. We first tested ATF6 activation using a chemical genetic method involving the expression in patient fibroblasts of a fusion protein composed of the constitutively active form of ATF6, ATF6(1-373)60, fused to a trimethoprim (TMP)-binding Escherichia coli DHFR-based destabiliz- 
ing domain ${ }^{31,61,62}$. This approach enables TMP-dependent activation of the ATF6 transcriptional pathway (Figure S2a). We transduced fibroblasts with DHFR.ATF6(1-373)- or DHFR.YFP-encoding, replication-incompetent adenoviruses, and then treated with TMP. We observed selective, TMP-dependent upregulation of strongly ATF6-dependent transcriptional targets ${ }^{31}$, such as HSPA5 and HSP90B1, with minimal induction of other UPR targets (Figure S2b), consistent with successful activation of the ATF6 transcriptional response. However, ATF6 activation had no detectable effect on collagen-I secretion from either WT or G425S $\alpha 1$ (I)-expressing primary fibroblasts (Figure S2c).

We next asked whether stress-independent induction of the IRE1-XBP1s transcriptional response might prove beneficial. For this experiment, we employed replication-incompetent adenoviruses encoding the active, spliced form of XBP1 (Ad.XBP1s) ${ }^{63}$ or encoding GFP (Ad.GFP) as a control. We observed that forced expression of XBP1s substantially increased $\alpha 1$ (I) secretion up to $\sim 300 \%$ from basal levels (with sensitivity to the exact Ad.XBP1s MOI used) from G425S $\alpha 1$ (I) patient primary fibroblasts (Figure 3a, Upper panels). Notably, the effect was selective specifically to fibroblasts that express mutant collagen-I. $\alpha 1$ (I) secretion by WT $\alpha 1$ (I)-expressing primary fibroblasts was not influenced by XBP1s expression. qPCR analysis confirmed selective induction of strongly XBP1s-dependent gene transcripts upon Ad.XBP1s transduction, as opposed to global UPR activation. As shown in Figure 3b, transcripts such as DNAJB9 were upregulated to levels near those attained by UPR activation using the ER stressor $\mathrm{Tg}^{31}$. In contrast, strongly ATF6-dependent genes, such as HSPA5 and HSP9OB1, or strongly ATF4-dependent genes, such as DDIT3 and PP1R15A, were upregulated to a much smaller extent compared to the effects of Tg treatment ${ }^{31,64 .}$

To further evaluate this phenomenon, we transduced fibroblasts with a wide range of Ad.XBP1s MOls to control the extent of activation of the XBP1s-dependent transcriptional response. We observed a strongly Ad.XBP1s dose-dependent increase in collagen-I secretion by the G425S $\alpha 1$ (I) primary patient fibroblasts (Figure 3a, Lower right panel; see Figure S3 for qPCR assessment of UPR activation). In contrast, collagen-I secretion from WT $\alpha 1(\mathrm{I})$ primary fibroblasts did not increase across a wide range of Ad.XBP1s doses (Figure 3a, Lower left panel). Notably, for both WT and G425S primary fibroblasts, collagen-I secretion began to drop once cells were transduced with very high doses of Ad.XBP1s. We have previously observed that tuning the level of expression of UPR transcription factors prevents any resultant cytotoxicity or global UPR activation 
caused by overloading the $\mathrm{ER}^{31}$. Consistent with this observation, Ad.XBP1s MOls that maximize induction of strongly XBP1s-dependent genes, such as DNAJB9, while minimizing upregulation of genes that are also strongly regulated by ATF6, such as HSPA5 (see Figure S3), resulted in optimal improvements of collagen-I secretion by G425S $\alpha 1$ (I) primary patient fibroblasts.

\section{XBP1s Pathway Activation Likely Restores G425S a1(I) Trafficking by Selectively Enhancing Mutant Collagen-I Folding}

We sought to gain initial insights into the underlying mechanism(s) that drive(s) an XBP1s-dependent selective increase in collagen-I secretion only from G425S $\alpha 1$ (I) OI-patient primary fibroblasts, but not from WT $\alpha 1$ (I) primary fibroblasts. We first used RNA-Seq to evaluate whether the transcriptional consequences of Ad.XBP1s treatment were substantively different in WT $\alpha 1(\mathrm{I})$-expressing versus $\mathrm{G} 425 \mathrm{~S} \alpha 1(\mathrm{I})$-expressing primary fibroblasts (Table S1). Correlation analysis revealed that the transcriptional remodeling caused by Ad.XBP1s was broadly similar across these two cell lines (Figure 4a). Moreover, gene set enrichment analysis $^{40}$ confirmed that Ad.XBP1s treatment upregulated expected ER proteostasis-related gene sets, with strongly enriched gene sets shared across both samples and the top five enriched gene sets being identical (Table S2; see Figure S4 for images of relevant enrichment plots). Thus, it seems unlikely that the selective increase in collagen-I secretion only from the G425S $\alpha 1(\mathrm{I})$ OI patient fibroblasts can be attributed to broadly different transcriptional consequences of XBP1s activation in those cells.

We next asked whether XBP1s might be selectively improving G425S $\alpha 1(\mathrm{I})$ trafficking by influencing cell growth, collagen-I transcription, global protein translation, or collagen-I translation specifically in the primary OI patient fibroblasts but not in the healthy donor control fibroblasts. Quantification of cellular ATP using a CellTiter-Gloß assay showed that Ad.XBP1s treatment had no detectable influence on cell growth rates in either G425S or WT $\alpha 1(\mathrm{I})$ primary fibroblasts (Figure 4b). Targeted qPCR analysis of collagen-I transcripts indicated that Ad.XBP1s treatment also did not substantially alter COL1A1 or COL1A2 mRNA levels in either cell line (Figure 4c). Finally, we used ${ }^{35}$ S-Cys/Met metabolic labeling experiments to evaluate possible effects on protein translation that might have occurred only in G425S $\alpha 1(\mathrm{I})$ primary patient fibroblasts. After labeling all nascent chains synthesized in a 10-minute span using ${ }^{35} \mathrm{~S}-\mathrm{Cys} /$ Met treatment, we immediately lysed the cells 
and separated the proteins using SDS-PAGE (Figure 4d). Quantification of the resulting autoradiographs revealed that global protein synthesis was significantly but insubstantially increased in WT $\alpha 1(\mathrm{I})$ fibroblasts upon treatment with Ad.XBP1s, while protein synthesis in G425S $\alpha 1(\mathrm{I})$ fibroblasts remained unchanged (Figure 4e). Collagenase digestion of the cell lysates allowed us to identify the procollagen-I bands on these SDS-PAGE gels, as indicated by the arrows in Figure 4d. Specifically quantifying the procollagen-I bands indicated that Ad.XBP1s treatment very modestly upregulated collagen-I synthesis in G425S $\alpha 1(\mathrm{I})$ and possibly in WT $\alpha 1(\mathrm{I})$ fibroblasts (Figure 4f).

Based on these observations, the ability of Ad.XBP1s treatment to selectively enhance collagen-I trafficking up to $\sim 300 \%$ in G425S $\alpha 1$ (I) primary fibroblasts but not in WT $\alpha 1(\mathrm{I})$ primary fibroblasts cannot likely be attributed to differential consequences of Ad.XBP1s for the overall cellular transcriptome, cell growth, collagenI transcription, or global protein translation. Given the effects observed, we would not completely rule out the possibility that modulation of collagen-I synthesis by Ad.XBP1s is involved. It appears more likely, however, that XBP1s-mediated remodeling of the ER proteostasis network selectively modulates downstream steps in collagen-I folding, quality control, or trafficking that are defective in the G425S $\alpha 1$ (I)-producing primary patient fibroblasts.

\section{XBP1s Activation Increases the Ratio of G425S Mutant to WT $\alpha 1$ (I) Polypeptides Secreted in the Form} of Stable Collagen Triple Helices

To further improve our understanding of how Ad.XBP1s selectively promotes collagen-I secretion from G425S $\alpha 1(\mathrm{I})$-expressing but not WT $\alpha 1(\mathrm{I})$-expressing primary fibroblasts, we sought to assess whether Ad.XBP1s alters the stability or composition of secreted collagen-I. This clinically severe form of OI is an autosomal dominant disease, and G425S patient fibroblasts synthesize both WT and mutant $\alpha 1(\mathrm{I})$. If Ad.XBP1s treatment does indeed enhance the folding or assembly of G425S $\alpha 1(\mathrm{I})$ into triple-helical collagen, we would predict that the enhanced collagen-I secretion observed would result in a higher ratio of G425S:WT $\alpha 1(\mathrm{I})$ in the secreted collagen-I obtained from Ad.XBP1s-transduced primary fibroblasts relative to Ad.GFP-transduced fibroblasts. 
To first evaluate whether Ad.XBP1s enhances the secretion of properly folded triple-helical collagen-I versus badly misfolded and/or non-triple-helical collagen-I from G425S $\alpha 1$ (I)-expressing primary patient fibroblasts, we turned to proteolysis assays. One characteristic of the fibrillar collagens is that stable, folded triple helices are resistant to protease digestion ${ }^{42,65}$. In contrast, denatured or badly misfolded collagen-I is susceptible to cleavage by pepsin and trypsin/chymotrypsin. We performed two separate sets of assays to assess the consequences of Ad.XBP1s treatment for the triple-helical structure of procollagen-I secreted from G425S $\alpha 1(\mathrm{I})$ primary patient fibroblasts. In the first assay, procollagen-I precipitated from media fractions was treated with trypsin or chymotrypsin and the extent of degradation was analyzed by immunoblotting with detection using a polyclonal antibody raised against the triple-helical domain of $\alpha 2(\mathrm{I})$. We found that, in the context of either Ad.GFP or Ad.XBP1s transduction, procollagen-I samples harvested from WT and G425S $\alpha 1$ (I)-producing primary fibroblasts were stable to prolonged treatment with trypsin and chymotrypsin to similar extents (Figure 5a; note that trypsin and chymotrypsin cleave the collagen-I propeptide domains causing the observed drop in molecular weight), consistent with Ad.XBP1s having no negative effects on the extent to which collagen-I attained a stable, triple-helical form.

In the second assay, we sought to assess whether and how Ad.XBP1s treatment altered the extent to which G425S $\alpha 1(\mathrm{I})$ was incorporated into the secreted collagen-I triple helices. Briefly, media from G425S $\alpha 1(\mathrm{I})$-expressing primary fibroblasts transduced with either Ad.GFP as a control or Ad.XBP1s was collected. Samples were treated with pepsin to degrade non-collagenous proteins and any non-triple-helical collagen, and then the secreted triple-helical collagen-I was precipitated and resolubilized. Separation of the digested protein samples using SDS-PAGE revealed the presence of pepsin-stable $\alpha 1(\mathrm{I})$ and $\alpha 2(\mathrm{I})$ polypeptides isolated by this protocol (Figure 5b), further confirming that Ad.XBP1s treatment does not result in excessive production of badly misfolded or unfolded collagen-I from G425S $\alpha 1(\mathrm{I})$ primary patient fibroblasts. We next applied a well-established MS protocol ${ }^{66-69}$ to evaluate whether XBP1s alters the ratio of G425S $\alpha 1(\mathrm{I})$ :WT $\alpha 1$ (I) secreted in a pepsin-stable, triple-helical form. LC-MS/MS analysis of the composition of the $\alpha 1(\mathrm{I})$ band from Figure $5 \mathbf{b}$ showed that, in media samples obtained from Ad.GFP-treated G425S $\alpha 1$ (I) primary fibroblasts, the G425S allelic product constituted roughly $20 \%$ of the total $\alpha 1(\mathrm{I})$ produced. Ad.XBP1s treatment doubled the fraction of the G425S allelic product present, shifting the ratio of G425S:WT $\alpha 1$ (I) to nearly 1:1 (Figure 5c). This increase 
bioRxiv preprint doi: https://doi.org/10.1101/2021.04.15.439909; this version posted April 16, 2021. The copyright holder for this preprint (which was not certified by peer review) is the author/funder. All rights reserved. No reuse allowed without permission.

in secretion of the G425S $\alpha 1(\mathrm{I})$ allelic product confirms that Ad.XBP1s treatment specifically promotes the folding and/or trafficking of the mutant collagen-I produced by the G425S $\alpha 1(\mathrm{I})$ OI patient primary fibroblasts. 


\section{DISCUSSION}

The G425S $\alpha 1(\mathrm{I})$ variant causes a severe, autosomal dominant form of $\mathrm{OI}^{44}$. The detailed origin of pathology is unclear, and may involve both intracellular and extracellular defects. Like many other Ol-causing variants $^{15,16,45-49}$, the G425S amino acid substitution results in a substantial collagen-I folding/secretion defect ${ }^{51}$. Such a proteostasis defect might also be expected to lead to cellular dysfunction, reducing overall bone health and motivating an effort to discover strategies to improve collagen-I trafficking in this genotype.

We find that collagen-I secretion by G425S $\alpha 1(\mathrm{I})$-expressing primary patient fibroblasts can be rescued by UPR arm-specific, ER stress-independent induction of the XBP1s transcriptional response. This effect is unique to XBP1s, as neither induction of the global UPR using small molecule stressors nor arm-selective, stress-independent induction of the ATF6 transcriptional pathway of the UPR has any beneficial effect. While

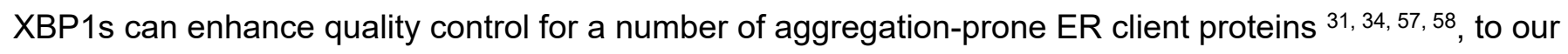
knowledge this observation constitutes the first example of XBP1s-mediated ER proteostasis network remodeling resolving a specific protein secretion defect.

Remarkably, despite similar transcriptional effects in both G425S $\alpha 1$ (I)-expressing primary patient fibroblasts and healthy donor primary fibroblasts, the consequences of XBP1s activity are highly selective for mutant collagen-I. That is, only the patient fibroblasts displayed an XBP1s-dependent increase in collagen-I secretion. Collagen-I secretion from WT fibroblasts was unaltered by XBP1s treatment. The effect appears most likely to be post-translational, perhaps involving improved chaperone-mediated folding and assembly of the mutant collagen-I. This notion is consistent with our analysis of the composition of the triple-helical collagen-I secreted upon XBP1s treatment, in which secretion of the mutant G425S $\alpha 1(\mathrm{I})$ allele is selectively enhanced relative to the WT $\alpha 1(\mathrm{I})$ allele.

Despite decades of research, current therapeutic strategies to address OI still have many deficits ${ }^{70,71 .}$ The most common clinical approach involves treatment with anti-resorptive agents, most prominently the bisphosphonates ${ }^{71}$. In contrast to inhibitors of catabolism, inducers of bone anabolism have recently received increasing attention. Of particular significance, increasing bone production via enhancing LRP5 signaling genetically or by targeting sclerostin and using antibodies to inhibit TGF $\beta$ signaling have both shown promise, 
with the TGF $\beta$-targeting strategy currently in clinical trials ${ }^{54,70,72,73}$. Prior work has also suggested the potential of the chemical chaperone 4-phenylbutyric acid to ameliorate skeletal defects in an Ol zebrafish model ${ }^{74,75}$.

The data presented here suggest that ER stress-independent IRE1-XBP1s activation may offer a new anabolism-targeted strategy to consider - one predicated on a beneficial remodeling of the ER proteostasis network to promote collagen-I folding and secretion, restore cell health, and potentially promote bone production. There is much work to be done following this initial in vitro proof-of-principle. Although primary fibroblasts are a highly relevant cell type that natively produces large quantities of collagen-I, and even though skin defects are commonly associated with $\mathrm{Ol}^{3,76}$, it will be important to evaluate the consequences of XBP1s activity in bone-producing osteoblasts. Unfortunately, primary osteoblasts are not widely available for this or many other OI variants. New OI animal models that incorporate prototypical Ol-causing collagen-I triple-helix mutations, such as Gly $\rightarrow$ Ser-inducing mutations in $C O L 1 A 1$, would be particularly beneficial in this regard and are currently lacking. The only triple helix-domain single amino acid substitution-based mouse models for OI that have been reported to date are the $\mathrm{G} 349 \mathrm{C} \alpha 1(\mathrm{I})$ and the $\mathrm{G} 610 \mathrm{C} \alpha 2(\mathrm{I})$ mice ${ }^{77,78}$. Further, it will be important to examine the scope of OI mutants for which XBP1s activation is beneficial. As with other strategies for Ol amelioration, it is likely that the approach is applicable to only a subset of variants. Progress will also benefit from improved small molecule-based IRE1-XBP1s activators, as current best-in-class compounds provide only short-term induction of the pathway and it is currently unclear whether they would function in osteoblasts ${ }^{79}$. Molecular-level mechanistic understanding of XBP1s-enhanced assembly and secretion would also be valuable, and will benefit from an improved understanding of fundamental collagen secretion and quality control mechanisms ${ }^{80-85}$. Such understanding could allow an even more specific strategy to resolve collagen proteostasis defects that does not rely upon large-scale XBP1s-mediated remodeling of the ER proteostasis network.

In conclusion, we have demonstrated that XBP1s-mediated enhancement of the ER proteostasis network can selectively improve the folding and secretion of an Ol-causing collagen-I variant in primary patient fibroblasts. Considering the vast array of mutations that engender collagen proteostasis defects and cause currently incurable collagenopathies, this finding should catalyze further efforts to understand and test the broad potential of proteostasis network modulation in these disorders. 


\section{ACKNOWLEDGEMENTS}

This work was supported by the National Institutes of Health (Grant 1R01AR071443), a Research Grant from the G. Harold and Leila Y. Mathers Foundation, a Dreyfus Foundation Teacher-Scholar Award, and an MIT International Science and Technology Initiatives grant (all to M.D.S.), and by the National Health and Medical Research Council of Australia (Grant GNT 1144807 to J.F.B. and S.R.L.) and the Victorian Government's Operational Infrastructure Support Program. A.S.D. was supported by a NIH Ruth L. Kirschstein predoctoral fellowship (F31AR067615). N.-D.D. was supported by Canadian Institutes of Health Research and Fonds de Recherche du Québec-Santé postdoctoral fellowships. A.A.B. was supported by the Johnson and Johnson UROP Scholar Program. Additional support was provided by an NIEHS grant (P30-ES002109) to the MIT Center for Environmental Health Sciences.

\section{NOTES}

The authors declare no competing financial interest. 


\section{REFERENCES}

1. Wong, M.Y. \& Shoulders, M.D. Targeting defective proteostasis in the collagenopathies. Curr. Opin. Chem. Biol. 50, 80-88 (2019).

2. Bateman, J.F., Boot-Handford, R.P. \& Lamandé, S.R. Genetic diseases of connective tissues: Cellular and extracellular effects of ECM mutations. Nat. Rev. Genet. 10, 173-183 (2009).

3. Marini, J.C. et al. Osteogenesis imperfecta. Nat. Rev. Dis. Primers. 3, e17052 (2017).

4. Marini, J.C. et al. Consortium for osteogenesis imperfecta mutations in the helical domain of type I collagen: regions rich in lethal mutations align with collagen binding sites for integrins and proteoglycans. Hum. Mutat. 28, 209-221 (2007).

5. Shoulders, M.D. \& Raines, R.T. Collagen structure and stability. Annu. Rev. Biochem. 78, 929-958 (2009).

6. Bella, J., Eaton, M., Brodsky, B. \& Berman, H. Crystal and molecular structure of a collagen-like peptide at 1.9 A resolution. Science. $\mathbf{2 6 6}, \mathbf{7 5 - 8 1}(1994)$.

7. Torre-Blanco, A., Adachi, E., Romanic, A.M. \& Prockop, D.J. Copolymerization of normal type I collagen with three mutated type I collagens containing substitutions of cysteine at different glycine positions in the $\alpha 1$ (I) chain. J. Biol. Chem. 267, 4968-4973 (1992).

8. Brenner, R.E., Vetter, U., Stöss, H., Müller, P.K. \& Teller, W.M. Defective collagen fibril formation and mineralization in osteogenesis imperfecta with congenital joint contractures (Bruck syndrome). Eur. J. Pediatr. 152, 505-508 (1993).

9. Culbert, A.A. et al. Substitutions of aspartic acid for glycine-220 and of arginine for glycine-664 in the triple helix of the proa1(I) chain of type I procollagen produce lethal osteogenesis imperfecta and disrupt the ability of collagen fibrils to incorporate crystalline hydroxyapatite. Biochem. J. 311, 815-820 (1995).

10. Sweeney, S.M. et al. Candidate cell and matrix interaction domains on the collagen fibril, the predominant protein of vertebrates. J. Biol. Chem. 283, 21187-21197 (2008).

11. An, B., Lin, Y.-S. \& Brodsky, B. Collagen interactions: Drug design and delivery. Adv. Drug. Deliv. Rev. 97, 69-84 (2016). 
12. Ben Amor, I.M., Roughley, P., Glorieux, F.H. \& Rauch, F. Skeletal clinical characteristics of osteogenesis imperfecta caused by haploinsufficiency mutations in COL1A1. J. Bone. Miner. Res. 28, 2001-2007 (2013).

13. Willing, M.C., Deschenes, S.P., Slayton, R.L. \& Roberts, E.J. Premature chain termination is a unifying mechanism for COL1A1 null alleles in osteogenesis imperfecta type I cell strains. Am. J. Hum. Genet. 59, 799-809 (1996).

14. Herbage, D., Borsali, F., Buffevant, C., Flandin, F. \& Aguercif, M. Composition, cross-linking and thermal stability of bone and skin collagens in patients with osteogenesis imperfecta. Metab. Bone Dis. Relat. Res. 4, 95-101 (1982).

15. Forlino, A. et al. Phenotypic comparison of an osteogenesis imperfecta Type IV proband with ade Novo 2 (I) Gly922 $\rightarrow$ Ser substitution in type I collagen and an unrelated patient with an identical mutation. Biochem. Mol. Med. 62, 26-35 (1997).

16. Bonadio, J. \& Byers, P.H. Subtle structural alterations in the chains of type I procollagen produce osteogenesis imperfecta type II. Nature. 316, 363-366 (1985).

17. Brinckmann, J. et al. Overhydroxylation of lysyl residues is the initial step for altered collagen crosslinks and fibril architecture in fibrotic skin. J. Invest. Dermatol. 113, 617-621 (1999).

18. Mirigian, L.S. et al. Osteoblast malfunction caused by cell stress response to procollagen misfolding in ג2(I)-G610C mouse model of osteogenesis imperfecta. J. Bone. Miner. Res. 31, 1608-1616 (2016).

19. Makareeva, E., Aviles, N.A. \& Leikin, S. Chaperoning osteogenesis: new protein-folding disease paradigms. Trends Cell Biol. 21, 168-176 (2011).

20. Lisse, T.S. et al. ER stress-mediated apoptosis in a new mouse model of osteogenesis imperfecta. PLoS Genet. 4, e7 (2008).

21. Tabas, I. \& Ron, D. Integrating the mechanisms of apoptosis induced by endoplasmic reticulum stress. Nat. Cell. Biol. 13, 184-190 (2011).

22. Walter, P. \& Ron, D. The unfolded protein response: from stress pathway to homeostatic regulation. Science. 334, 1081-1086 (2011).

23. Wong, M.Y. et al. Adapting secretory proteostasis and function through the Unfolded Protein Response. Curr. Top. Microbiol. Immunol. 414, 1-25 (2017). 
24. Bartoszewski, R. et al. Activation of the unfolded protein response by $\triangle$ F508 CFTR. Am. J. Respir. Cell. Mol. Biol. 39, 448-457 (2008).

25. Plate, L. \& Wiseman, R.L. Regulating secretory proteostasis through the unfolded protein response: from function to therapy. Trends. Cell. Biol. 27, 722-737 (2017).

26. Boot-Handford, R.P. \& Briggs, M.D. The unfolded protein response and its relevance to connective tissue diseases. Cell Tissue Res. 339, 197-211 (2009).

27. Chessler, S.D. \& Byers, P.H. Bip binds type-I procollagen Pro $\alpha$ Chains with mutations in the carboxylterminal propeptide synthesized by cells from patients with osteogenesis imperfecta. J. Biol. Chem. 268, 18226-18233 (1993).

28. Forlino, A., Kuznetsova, N.V., Marini, J.C. \& Leikin, S. Selective retention and degradation of molecules with a single mutant $\alpha 1(\mathrm{I})$ chain in the Brtl IV mouse model of OI. Matrix Biol. 26, 604-614 (2007).

29. Blackwood, E.A. et al. Pharmacologic ATF6 activation confers global protection in widespread disease models by reprograming cellular proteostasis. Nat. Commun. 10, e187 (2019).

30. Martinez, A., Lopez, N., Gonzalez, C. \& Hetz, C. Targeting of the unfolded protein response (UPR) as therapy for Parkinson's disease. Biol. Cell. 111, 161-168 (2019).

31. Shoulders, Matthew D. et al. Stress-independent activation of XBP1s and/or ATF6 reveals three functionally diverse ER proteostasis environments. Cell Rep. 3, 1279-1292 (2013).

32. Waldherr, S.M., Strovas, T.J., Vadset, T.A., Liachko, N.F. \& Kraemer, B.C. Constitutive XBP-1smediated activation of the endoplasmic reticulum unfolded protein response protects against pathological tau. Nat. Commun. 10, e4443 (2019).

33. Brodsky, J.L. et al. Remodeling the endoplasmic reticulum proteostasis network restores proteostasis of pathogenic GABAA receptors. PLoS One. 13, e0207948 (2018).

34. Cooley, C.B. et al. Unfolded protein response activation reduces secretion and extracellular aggregation of amyloidogenic immunoglobulin light chain. Proc. Natl. Acad. Sci. USA. 111, 1304613051 (2014).

35. Mu, T.-W. et al. Chemical and biological approaches synergize to ameliorate protein-folding diseases. Cell. 134, 769-781 (2008). 
36. Ong, Derrick Sek T. et al. FKBP10 depletion enhances glucocerebrosidase proteostasis in Gaucher disease fibroblasts. Chem. Biol. 20, 403-415 (2013).

37. Dobin, A. et al. STAR: ultrafast universal RNA-seq aligner. Bioinformatics. 29, 15-21 (2013).

38. Derr, A. et al. End sequence analysis toolkit (ESAT) expands the extractable information from singlecell RNA-seq data. Genome Res. 26, 1397-1410 (2016).

39. Love, M.I., Huber, W. \& Anders, S. Moderated estimation of fold change and dispersion for RNA-seq data with DESeq2. Genome Biol. 15, 550 (2014).

40. Subramanian, A. et al. Gene set enrichment analysis: A knowledge-based approach for interpreting genome-wide expression profiles. Proc. Natl. Acad. Sci. USA. 102, 15545-15550 (2005).

41. Mootha, V.K. et al. PGC-1 $\alpha$-responsive genes involved in oxidative phosphorylation are coordinately downregulated in human diabetes. Nat. Genet. 34, 267-273 (2003).

42. Bruckner, P. \& Prockop, D.J. Proteolytic-enzymes as probes for the triple-helical conformation of procollagen. Anal. Biochem. 110, 360-368 (1981).

43. Makareeva, E., Cabral, W.A., Marini, J.C. \& Leikin, S. Molecular mechanism of $\alpha 1$ (I)-osteogenesis imperfecta/Ehlers-Danlos syndrome: unfolding of an $\mathrm{N}$-anchor domain at the $\mathrm{N}$-terminal end of the type I collagen triple helix. J. Biol. Chem. 281, 6463-6470 (2006).

44. Mackay, K., Byers, P.H. \& Dalgleish, R. An RT-PCR-SSCP screening strategy for detection of mutations in the gene encoding the $\alpha 1$ chain of type I collagen: application to four patients with osteogenesis imperfecta. Hum. Mol. Genet. 2, 1155-1160 (1993).

45. Lindert, U. et al. Insight into the pathology of a COL1A1 signal peptide heterozygous mutation leading to severe osteogenesis imperfecta. Calcif. Tissue Int. 102, 373-379 (2017).

46. Willing, M.C. et al. Heterozygosity for a large deletion in the $\alpha 2(\mathrm{I})$ collagen gene has a dramatic effect on type-I collagen secretion and produces perinatal lethal osteogenesis imperfecta. J. Biol. Chem. 263, 8398-8404 (1988).

47. Raghunath, M., Bruckner, P. \& Steinmann, B. Delayed triple helix formation of mutant collagen from patient with osteogenesis imperfecta. J. Mol. Biol. 236, 940-949 (1994). 
48. Lamandé, S.R. et al. Endoplasmic reticulum-mediated quality control of type I collagen production by cells from osteogenesis imperfecta patients with mutations in the pro 1 1(I) chain carboxyl-terminal propeptide which impair subunit assembly. J. Biol. Chem. 270, 8642-8649 (1995).

49. Bateman, J.F., Chan, D., Mascara, T., Rogers, J.G. \& Cole, W.G. Collagen defects in lethal perinatal osteogenesis imperfecta. Biochem. J. 240, 699-708 (1986).

50. Bateman, J.F., Mascara, T., Chan, D. \& Cole, W.G. Abnormal type I collagen metabolism by cultured fibroblasts in lethal perinatal osteogenesis imperfecta. Biochem. J. 217, 103-115 (1984).

51. Makareeva, E. et al. Structural heterogeneity of type I collagen triple helix and its role in osteogenesis imperfecta. J. Biol. Chem. 283, 4787-4798 (2008).

52. Sehgal, P. et al. Inhibition of the sarco/endoplasmic reticulum (ER) Ca2+-ATPase by thapsigargin analogs induces cell death via ER Ca2+ depletion and the unfolded protein response. J. Biol. Chem. 292, 19656-19673 (2017).

53. Kurn, N. \& Duksin, D. Effects of tunicamycin on protein glycosylation and development inVolvox carteri. Wilhelm Roux. Arch. Dev. Biol. 191, 169-175 (1982).

54. Grafe, I. et al. Excessive transforming growth factor- $\beta$ signaling is a common mechanism in osteogenesis imperfecta. Nat. Med. 20, 670-675 (2014).

55. Chiang, W.-C., Hiramatsu, N., Messah, C., Kroeger, H. \& Lin, J.H. Selective activation of ATF6 and PERK endoplasmic reticulum stress signaling pathways prevent mutant rhodopsin accumulation. Invest. Opthalmol. Vis. Sci. 53, 7159-7166 (2012).

56. Sebastian, R.M. \& Shoulders, M.D. Chemical biology framework to illuminate proteostasis. Annu. Rev. Biochem. 89, 529-555 (2020).

57. Valdes, P. et al. Control of dopaminergic neuron survival by the unfolded protein response transcription factor XBP1. Proc. Natl. Acad. Sci. USA. 111, 6804-6809 (2014).

58. Zuleta, A., Vidal, R.L., Armentano, D., Parsons, G. \& Hetz, C. AAV-mediated delivery of the transcription factor XBP1s into the striatum reduces mutant Huntingtin aggregation in a mouse model of Huntington's disease. Biochem. Biophys. Res. Commun. 420, 558-563 (2012).

59. Plate, L. et al. Quantitative interactome proteomics reveals a molecular basis for ATF6-dependent regulation of a destabilized amyloidogenic protein. Cell. Chem. Biol. 26, 913-925 (2019). 
60. Haze, K. et al. Mammalian transcription factor ATF6 is synthesized as a transmembrane protein and activated by proteolysis in response to endoplasmic reticulum stress. Mol. Biol. Cell. 10, 3787-3799 (1999).

61. Shoulders, M.D., Ryno, L.M., Cooley, C.B., Kelly, J.W. \& Wiseman, R.L. Broadly applicable methodology for the rapid and dosable small molecule-mediated regulation of transcription factors in human cells. J. Am. Chem. Soc. 135, 8129-8132 (2013).

62. Iwamoto, M., Björklund, T., Lundberg, C., Kirik, D. \& Wandless, T.J. A general chemical method to regulate protein stability in the mammalian central nervous system. Chem. Biol. 17, 981-988 (2010).

63. Calfon, M. et al. IRE1 couples endoplasmic reticulum load to secretory capacity by processing the XBP1 mRNA. Nature. 415, 92-96 (2002).

64. Grandjean, J.M.D. et al. Deconvoluting stress-responsive proteostasis signaling pathways for pharmacologic activation using targeted RNA sequencing. ACS Chem. Biol. 14, 784-795 (2019).

65. Pace, J.M., Kuslich, C.D., Willing, M.C. \& Byers, P.H. Disruption of one intra-chain disulphide bond in the carboxyl-terminal propeptide of the proa1(I) chain of type I procollagen permits slow assembly and secretion of overmodified, but stable procollagen trimers and results in mild osteogenesis imperfecta. $J$. Med. Genet. 38, 443-449 (2001).

66. Hudson, D.M. et al. P3h3-null and Sc65-null mice phenocopy the collagen lysine under-hydroxylation and cross-linking abnormality of Ehlers-Danlos syndrome type VIA. J. Biol. Chem. 292, 3877-3887 (2017).

67. Hudson, D.M., Archer, M., King, K.B. \& Eyre, D.R. Glycation of type I collagen selectively targets the same helical domain lysine sites as lysyl oxidase-mediated cross-linking. J. Biol. Chem. 293, 1562015627 (2018).

68. Kalamajski, S. et al. Increased C-telopeptide cross-linking of tendon type I collagen in fibromodulindeficient mice. J. Biol. Chem. 289, 18873-18879 (2014).

69. Hudson, D.M., Garibov, M., Dixon, D.R., Popowics, T. \& Eyre, D.R. Distinct post-translational features of type I collagen are conserved in mouse and human periodontal ligament. J. Periodontal Res. 52, 1042-1049 (2017). 
70. Kaupp, S. et al. Combination therapy in the Col1 22 G610C mouse model of Osteogenesis Imperfecta reveals an additive effect of enhancing LRP5 signaling and inhibiting TGF $\beta$ signaling on trabecular bone but not on cortical bone. Bone. 131, e115084 (2020).

71. Marom, R., Lee, Y.-C., Grafe, I. \& Lee, B. Pharmacological and biological therapeutic strategies for osteogenesis imperfecta. Am. J. Med. Genet. C. Semin. Med. Genet. 172, 367-383 (2016).

72. Jacobsen, C.M. et al. Targeting the LRP5 pathway improves bone properties in a mouse model of osteogenesis imperfecta. J. Bone Miner. Res. 29, 2297-2306 (2014).

73. Tauer, J.T., Abdullah, S. \& Rauch, F. Effect of Anti-TGF- $\beta$ treatment in a mouse model of severe osteogenesis imperfecta. J. Bone. Miner. Res. 34, 207-214 (2019).

74. Gioia, R. et al. The chaperone activity of 4PBA ameliorates the skeletal phenotype of Chihuahua, a zebrafish model for dominant osteogenesis imperfecta. Hum. Mol. Genet. 26, 2897-2911 (2017).

75. Besio, R. et al. 4-PBA ameliorates cellular homeostasis in fibroblasts from osteogenesis imperfecta patients by enhancing autophagy and stimulating protein secretion. Biochim. Biophys. Acta. Mol. Basis. Dis. 1864, 1642-1652 (2018).

76. Hansen, B. \& Jemec, G.B.E. The mechanical properties of skin in osteogenesis imperfecta. Arch. Dermatol. 138, 909-911 (2002).

77. Daley, E. et al. Variable bone fragility associated with an Amish COL1A2 variant and a knock-in mouse model. J. Bone. Miner. Res. 25, 247-261 (2010).

78. Forlino, A., Porter, F.D., Lee, E.J., Westphal, H. \& Marini, J.C. Use of the cre/lox recombination system to develop a non-lethal knock-in murine model for osteogenesis imperfecta with an $\alpha 1$ (I) G349C substitution. J. Biol. Chem. 274, 37923-37931 (1999).

79. Grandjean, J.M.D. et al. Pharmacologic IRE1/XBP1s activation confers targeted ER proteostasis reprogramming. Nat. Chem. Biol. 16, 1052-1061 (2020).

80. Omari, S. et al. Noncanonical autophagy at ER exit sites regulates procollagen turnover. Proc. Natl. Acad. Sci. USA. 115, E10099-E10108 (2018).

81. Wong, M.Y. et al. A high-throughput assay for collagen secretion suggests an unanticipated role for Hsp90 in collagen production. Biochemistry. 57, 2814-2827 (2018). 
82. Raote, I. et al. TANGO1 builds a machine for collagen export by recruiting and spatially organizing COPII, tethers and membranes. eLife. 7, e32723 (2018).

83. Forrester, A. et al. A selective ER-phagy exerts procollagen quality control via a calnexin-FAM 134B complex. EMBO J. 38, e99847 (2018).

84. Zhang, Z. et al. Broadly conserved roles of TMEM131 family proteins in intracellular collagen assembly and secretory cargo trafficking. Sci. Adv. 6, eaay7667 (2020).

85. DiChiara, A.S. et al. Mapping and exploring the collagen-I proteostasis network. ACS Chem Biol. 11, 1408-1421 (2016).

86. Berisio, R., Vitagliano, L., Mazzarella, L. \& Zagari, A. Crystal structure of the collagen triple helix model [(Pro-Pro-Gly)10]3. Protein. Sci. 11, 262-270 (2009).

87. Hayashi, T. \& Nagai, Y. The anomalous behavior of collagen peptides on sodium dodecyl sulfatepolyacrylamide gel electrophoresis is due to the low content of hydrophobic amino acid residues. $J$. Biochem. 87, 803-808 (1980). 


\section{FIGURE LEGEND}

\section{Figure 1}

High-resolution crystal structure of a collagen triple helix formed from (Pro-Pro-Gly) ${ }_{10}$ (PDB1K6F) ${ }^{86}$. An exemplary glycine to serine substitution and resulting hydrogen bond disruption is shown, obtained by replacing a single Gly residue with Ser. A diagram of the staggered arrangement of the three distinctive polypeptide strands in a segment of a triple helix and the ladder of interstrand hydrogen bonds that is disrupted by a Gly $\rightarrow$ Ser substitution is also shown.

\section{Figure 2}

a. Immunoblot showing secretion of $\alpha 1(\mathrm{I})$ and $\alpha 2(\mathrm{I})$ from WT $\alpha 1(\mathrm{I})$ and G425S $\alpha 1(\mathrm{I})$ primary patient fibroblasts. Equal numbers of cells were plated and collagen-I production was initiated by treatment with sodium ascorbate (Asc) for $24 \mathrm{~h}$. The presence of multiple $\alpha 1(\mathrm{I})$ bands corresponds to pro $\alpha 1(\mathrm{I})$ and $\alpha 1(\mathrm{I})$ before and after cleavage of the C-propeptide and/or the N-propeptide.

b. qPCR analysis of unfolded protein response (UPR)-regulated transcripts in WT $\alpha 1$ (I) and G425S $\alpha 1$ (I) primary fibroblasts. Fold-change in transcript levels was normalized to WT $\alpha 1$ (I) fibroblasts not treated with sodium ascorbate. Thapsigargin ( $\mathrm{Tg}$ ) treatment was used as a positive control for UPR activation. Error bars represent SD across three technical replicates.

c. Immunoblot showing secretion of $\alpha 1$ (I) from WT $\alpha 1(\mathrm{I})$ and G425S $\alpha 1$ (I) primary fibroblasts treated with increasing concentrations of Tg (top) or tunicamycin (Tm) (bottom). One day post-plating, cells were treated with $\mathrm{Tg}$ or $\mathrm{Tm}$ and $\alpha 1(\mathrm{I})$ secretion was initiated by treatment with Asc, followed by harvesting for analysis after $24 \mathrm{~h}$.

\section{Figure 3}

a. (Upper panels) Immunoblot showing secretion of $\alpha 1$ (I) from WT $\alpha 1$ (I) (top left panel) or G425S $\alpha 1$ (I) (top right panel) primary fibroblasts transduced with Ad.GFP or Ad.XBP1s. WT $\alpha 1(\mathrm{I})$ and G425S $\alpha 1$ (I) patient fibroblasts were transduced with replication-incompetent adenoviruses engineered to deliver a constitutively expressed XBP1s gene (Ad.XBP1s) or a constitutively expressed GFP gene (Ad.GFP). Transduction with Ad.GFP was used as a negative control. $\alpha 1(\mathrm{I})$ secretion was initiated by treatment of cells with sodium ascorbate (Asc) for $24 \mathrm{~h}$ prior to harvesting samples for analysis. The presence of multiple $\alpha 1(\mathrm{I})$ bands corresponds to pro $\alpha 1(\mathrm{I})$ and $\alpha 1(\mathrm{I})$ before and after cleavage of the C-propeptide and/or the N-propeptide. Lanes separated by a line were run on the same gel but were non-contiguous in that gel. (Lower panels) Dose-response immunoblots showing secretion of $\alpha 1(\mathrm{I})$ and $\alpha 2(\mathrm{I})$ from WT $\alpha 1$ (I) (bottom left panel) and G425S $\alpha 1$ (I) (bottom right panel) primary fibroblasts transduced with increasing volumes of Ad.XBP1s. Induction of collagen-I secretion was initiated by treatment of cells with ascorbate for $24 \mathrm{~h}$ prior to harvesting samples for analysis. b. qPCR analysis of UPR-dependent gene transcripts from cells treated as in the upper panels of Figure $\mathbf{3 a}$. Fold-change in transcript levels was normalized to WT $\alpha 1(\mathrm{I})$ fibroblasts not treated with sodium ascorbate. 
Thapsigargin (Tg) treatment was used as a positive control for UPR activation. Error bars represent SD across three technical replicates.

\section{Figure 4}

a. Correlation plot showing all transcripts expressed in sodium ascorbate-treated WT $\alpha 1(\mathrm{I})$ or G425S $\alpha 1$ (I) primary fibroblasts transduced with Ad.XBP1s versus Ad.GFP. Genes that were significantly upregulated $(>1.75-$ fold and Benjamin-Hochberg adjusted $p$-value $<0.05)$ in both WT $\alpha 1(\mathrm{I})$ and G425S $\alpha 1(\mathrm{I})$ upon treatment with Ad.XBP1s vs Ad.GFP are shown in blue.

b. Quantification of cellular ATP using the CellTiter-Glo® assay for sodium ascorbate-treated WT $\alpha 1$ (I) or G425S $\alpha 1$ (I) primary fibroblasts transduced with Ad.XBP1s versus Ad.GFP. Fold-change was normalized to Ad.GFP transductions. Error bars represent SD across three biological replicates.

c. qPCR analysis of COL1A1 and COL1A2 gene transcripts in sodium ascorbate-treated WT $\alpha 1(\mathrm{I})$ or G425S $\alpha 1$ (I) primary fibroblasts transduced with Ad.XBP1s versus Ad.GFP. Error bars represent SD across three biological replicates. Fold-change was normalized to WT $\alpha 1(\mathrm{I})$ cells treated with Ad.GFP.

d. Representative SDS-PAGE gel of ${ }^{35}$ S-Cys/Met-labeled cell lysates from sodium ascorbate-treated WT $\alpha 1$ (I) or G425S a1(I) primary fibroblasts transduced with Ad.XBP1s versus Ad.GFP. Samples were split in half and treated with or without bacterial collagenase for three days to determine which protein bands derived from col-

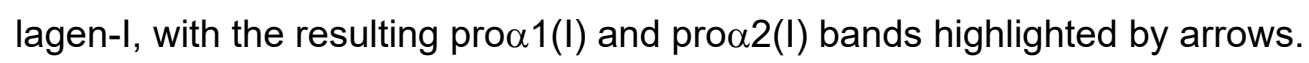

e. Quantification of the total ${ }^{35} \mathrm{~S}$ signal in the absence of collagenase for the samples in Figure 4d. Signal was normalized to cells treated with Ad.GFP for each cell line. Error bars represent SD over three biological replicates. * and n.s. correspond to $p$-value $<0.05$ and not significant, respectively.

f. Quantification of the collagen-I signal for the samples in Figure 4d. Signal was normalized to cells treated with Ad.GFP for each cell line. Error bars represent SD over three biological replicates. * and n.s. correspond to $p$-value $<0.05$ and not significant, respectively.

\section{Figure 5}

a. Immunoblot showing protease digestion of secreted $\alpha 1(\mathrm{I})$ and $\alpha 2(\mathrm{I})$ from WT $\alpha 1(\mathrm{I})$ or G425S $\alpha 1(\mathrm{I})$ primary fibroblasts transduced with Ad.GFP or Ad.XBP1s. Collagen-I was precipitated from the media and then treated with trypsin or chymotrypsin for $2 \mathrm{~h}$ at room temperature, following which the reaction was quenched prior to analysis by immunoblotting using a polyclonal antibody against the triple-helical domain of $\alpha 2(\mathrm{I})$.

b. Coomassie-stained SDS-PAGE gel showing pepsin-digested collagen-I secreted from sodium ascorbate (Asc)-treated WT $\alpha 1(\mathrm{I})$ or G425S $\alpha 1(\mathrm{I})$ primary fibroblasts transduced with Ad.GFP or Ad.XBP1s. Collagen-I was precipitated from media, resolubilized, and treated with pepsin. Note that $\alpha 1(\mathrm{I})$ is known to display lower electrophoretic mobility than $\alpha 2(\mathrm{I})^{87}$.

c. Representative MS1 scan highlighting the four relevant $\mathrm{m} / \mathrm{z}$ ratios and quantification of the WT $\alpha 1(\mathrm{I})$ and G425S $\alpha 1(\mathrm{I})$ allelic products secreted from G425S $\alpha 1(\mathrm{I})$ primary patient fibroblasts transduced with Ad.GFP or 
Ad.XBP1s. The $\alpha 1(\mathrm{I})$ band from Figure $5 \mathbf{b}$ was excised, reduced, alkylated, and trypsinized. The resultant peptides were analyzed by LC-MS/MS analysis to evaluate the abundance of the WT G425 versus mutant $\mathrm{S} 425 \alpha 1(\mathrm{I})$-containing peptides. $\mathrm{m} / \mathrm{z}$ ratios of 652.1 and 667.0 correspond to the peptide without lysine hydroxylation, while the ratios of 660.1 and 675.1 accounts for the hydroxylated lysine. The difference between the WT $\alpha 1(\mathrm{I})$ and G425S $\alpha 1(\mathrm{I})$ peptides was quantified by analysis of the area under the curve of the LC trace for each peptide. 
bioRxiv preprint doi: https://doi.org/10.1101/2021.04.15.439909; this version posted April 16, 2021. The copyright holder for this preprint (which was not certified by peer review) is the author/funder. All rights reserved. No reuse allowed without permission.

\section{Figure 1}

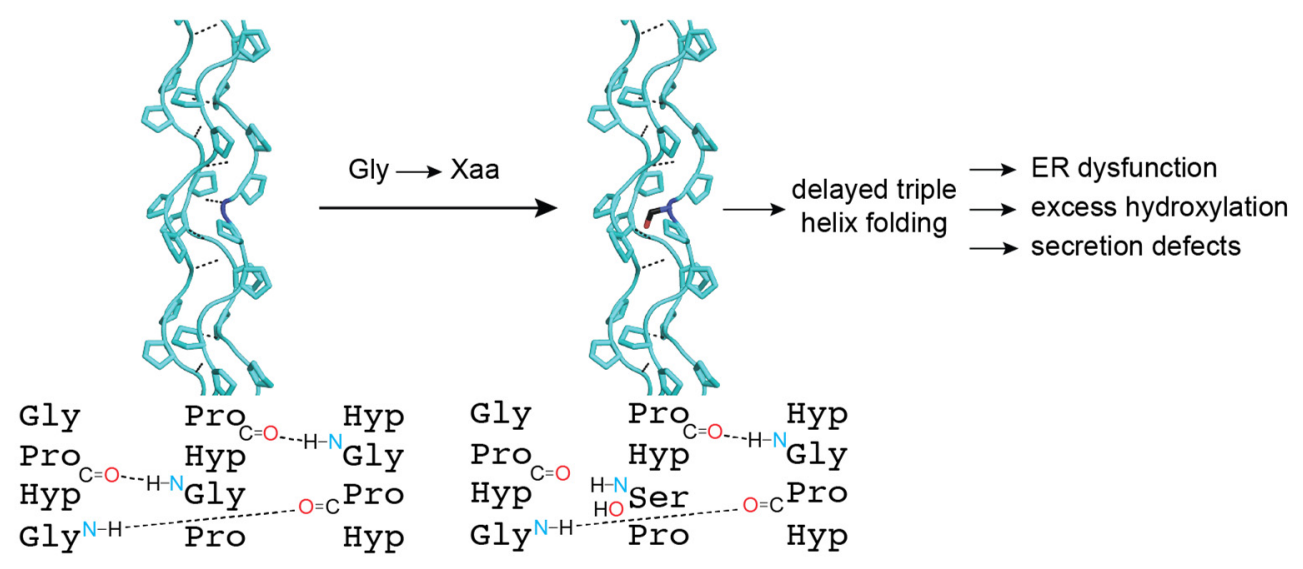


bioRxiv preprint doi: https://doi.org/10.1101/2021.04.15.439909; this version posted April 16, 2021. The copyright holder for this preprint (which was not certified by peer review) is the author/funder. All rights reserved. No reuse allowed without permission.

\section{Figure 2}

a

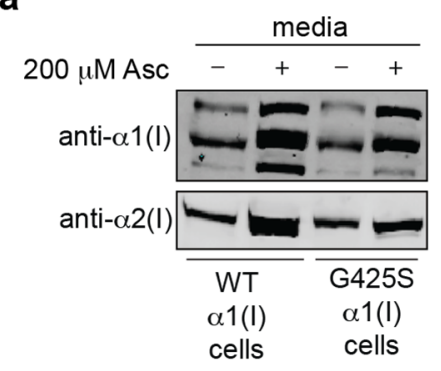

b

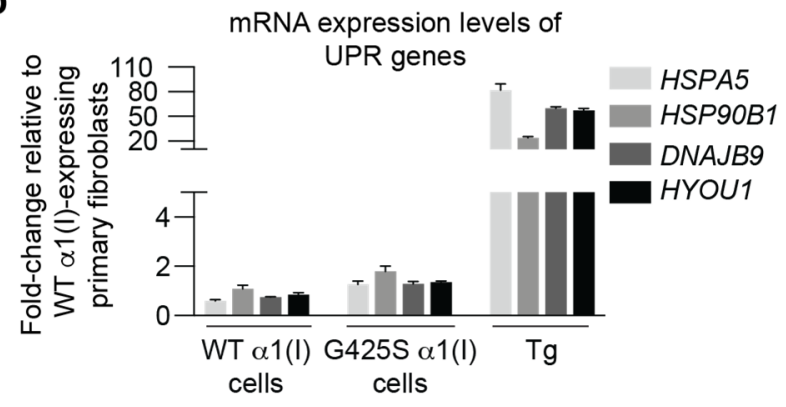

C
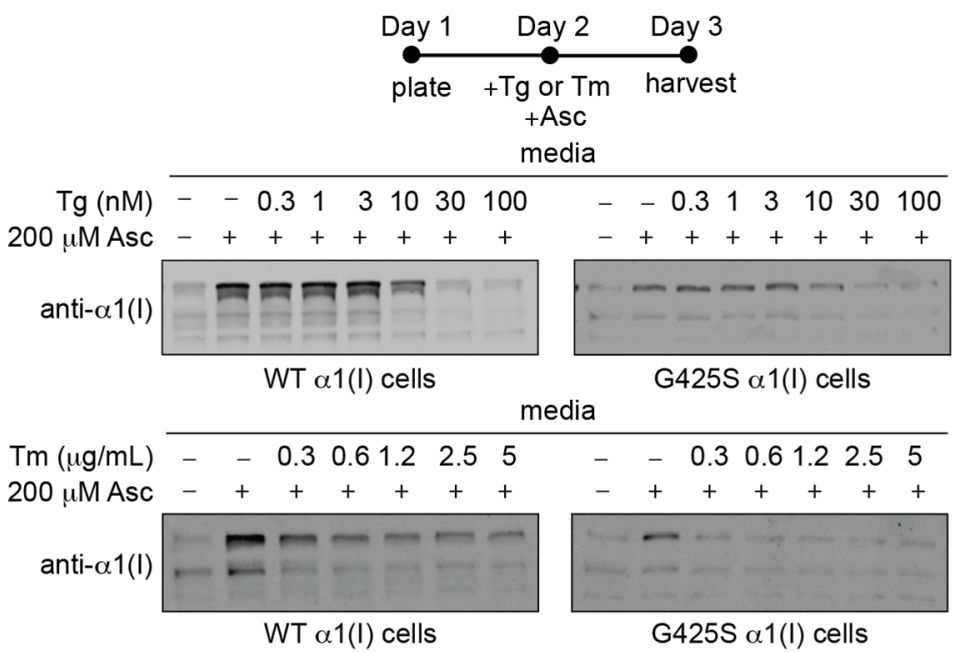
bioRxiv preprint doi: https://doi.org/10.1101/2021.04.15.439909; this version posted April 16, 2021. The copyright holder for this preprint (which was not certified by peer review) is the author/funder. All rights reserved. No reuse allowed without permission.

\section{Figure 3}

a
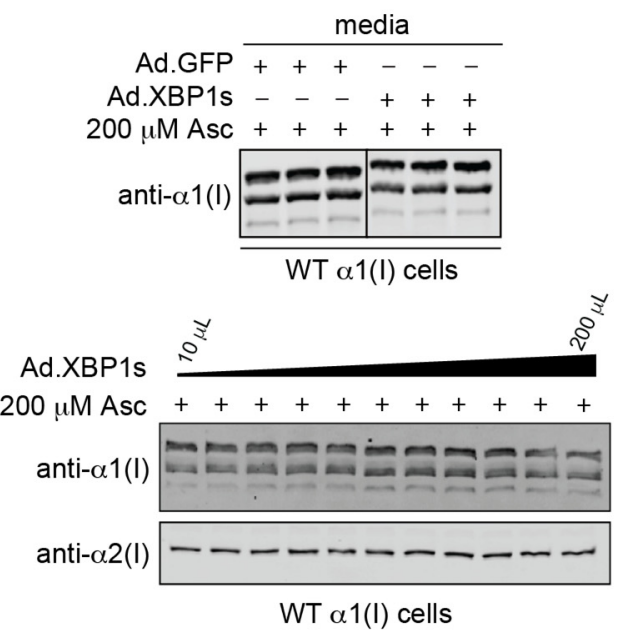

b

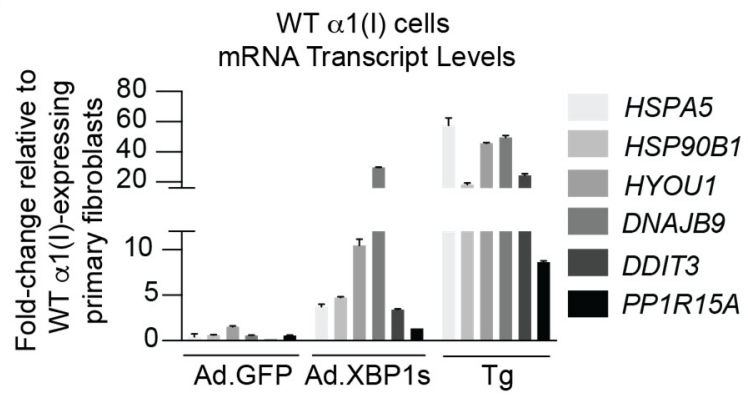

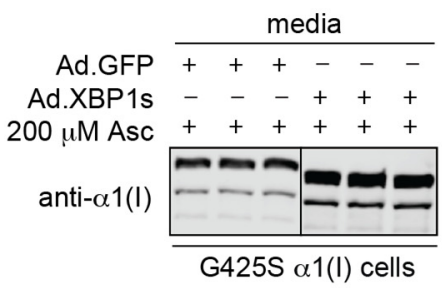

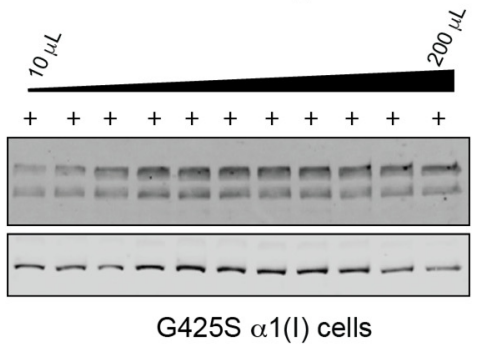

G425S $\alpha 1$ (I) cells mRNA Transcript Levels

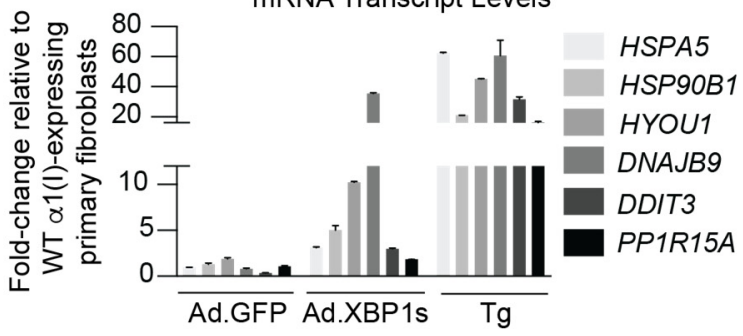


bioRxiv preprint doi: https://doi.org/10.1101/2021.04.15.439909; this version posted April 16, 2021. The copyright holder for this preprint (which was not certified by peer review) is the author/funder. All rights reserved. No reuse allowed without permission.

\section{Figure 4}

a

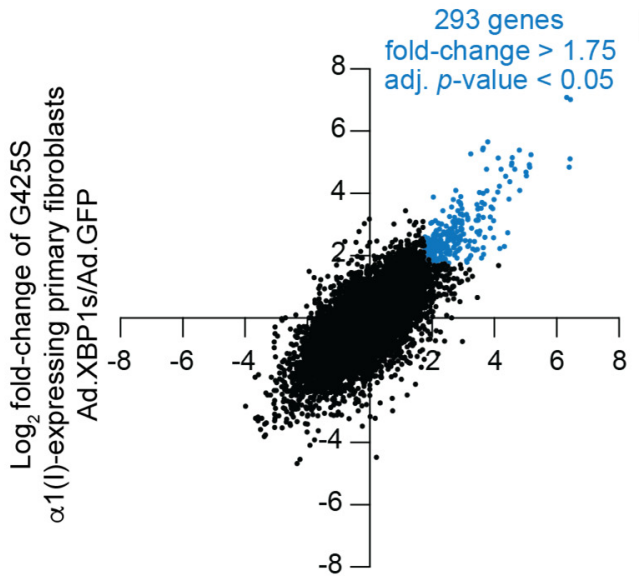

$\log _{2}$ fold-change of

WT $\alpha 1$ (I)-expressing fibroblasts Ad.XBP1s/Ad.GFP

d

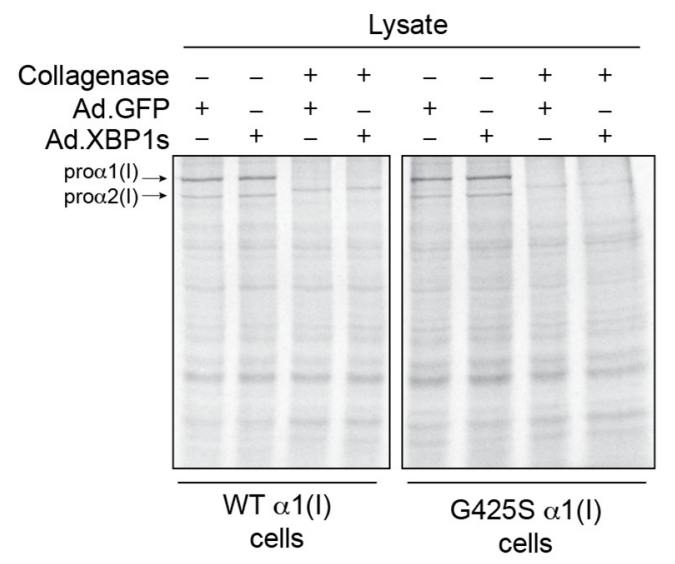

b

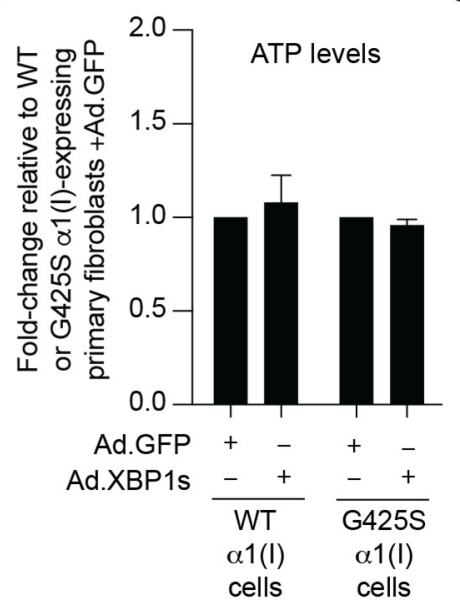

e

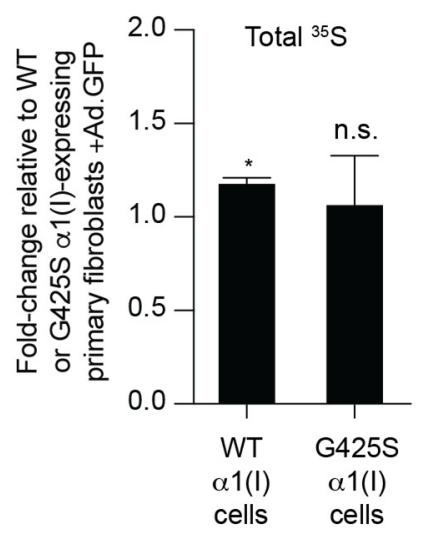

c

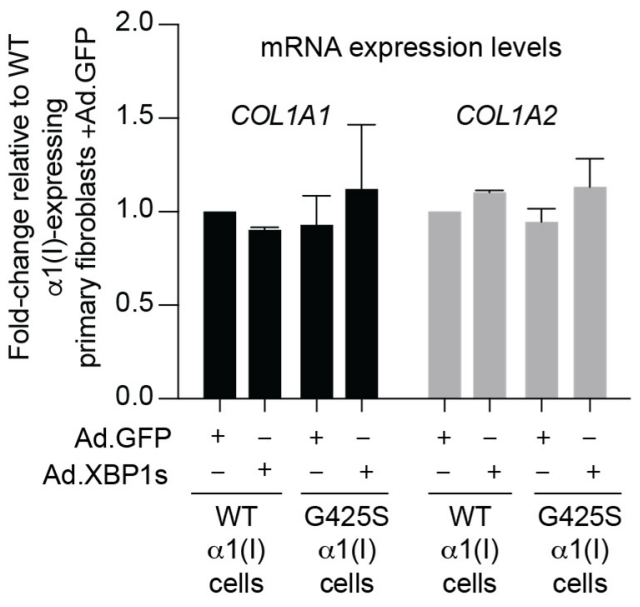

f

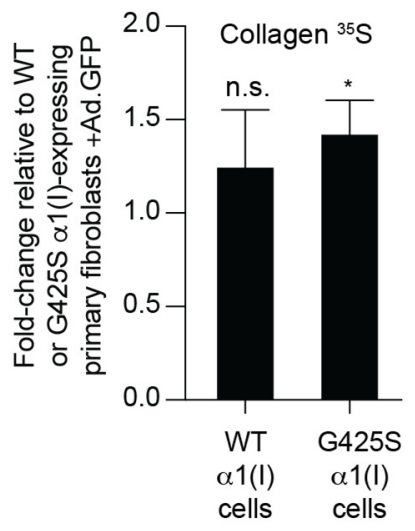




\section{Figure 5}

a

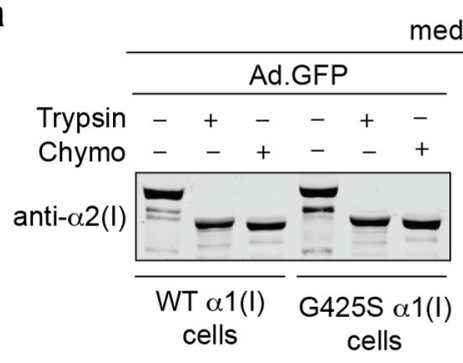

media

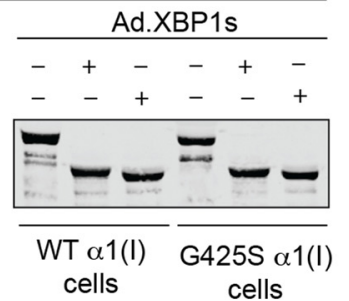

b

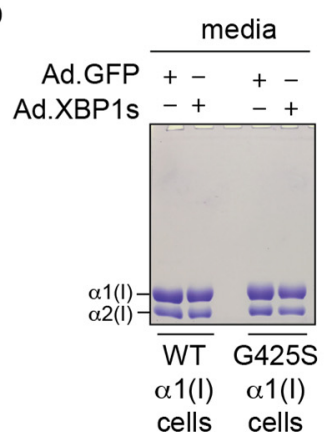

C

\section{Ad.GFP}

G425S $\alpha 1$ (I) cells

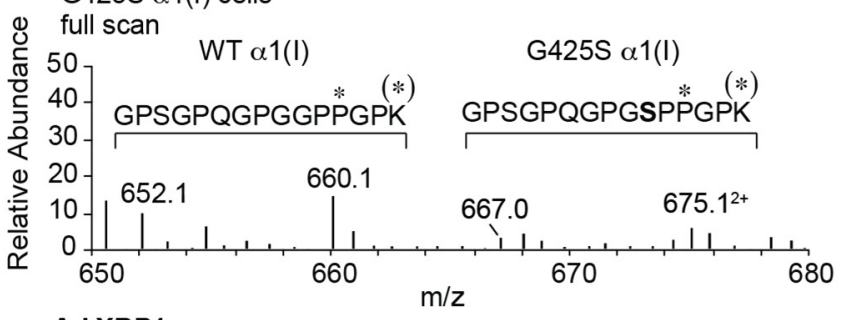

Ad.XBP1s

G425S $\alpha 1$ (I) cells

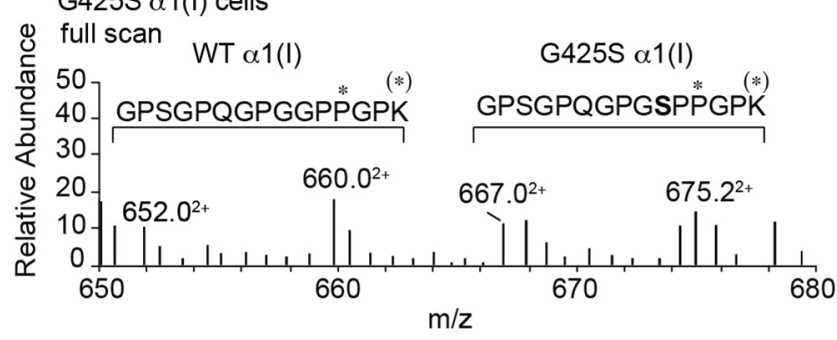

Ad.GFP

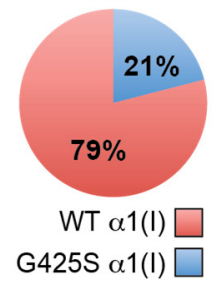

Ad.XBP1s

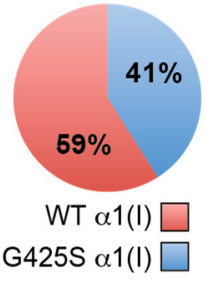

